\title{
Nuevo hallazgo de Miersia cornuta Phil. (Gilliesieae-Alliaceae)
}

\author{
New discovery of Miersia cornuta Phil. (Gilliesieae-Alliaceae)
}

\author{
Inelia Escobar ${ }^{1}$, Patricio Novoa $^{2}$, Eduardo Ruiz ${ }^{1}$, María Negritto $^{1}$ \& Carlos Baeza ${ }^{1}$ \\ ${ }^{1}$ Departamento de Botánica, Universidad de Concepción, Casilla 160-C, Concepción, Chile. \\ ${ }^{2}$ Departamento Horticultura, Jardín Botánico Nacional, Casilla 488, Viña del Mar, Chile. \\ iescobar@udec.cl
}

\begin{abstract}
A new discovery of Miersia cornuta Phil. from Valparaiso Region, Chile, is presented. Details of flower and fruit has been unclear, what has caused this taxon has been considered a doubtful species. The complete description, commentaries, new localities of this endemic species, and a key for Miersia species are given.
\end{abstract}

El género Miersia Lindl. endémico de Chile, posee cuatro especies, que crecen en la zona central desde los $31^{\circ} 54^{\prime \prime}$ a los $39^{\circ} 24^{\prime \prime}$ de latitud sur (Zuloaga et al. 2008, Escobar et al., datos no publicados).

R.A. Philippi (1873) describe Miersia cornuta sobre la base de materiales recolectados en la cuesta El Melón, Región de Valparaíso, en septiembre de 1865. En la descripción se señala que $M$. cornuta presenta hojas de a lo menos 32,5 cm de largo, dos veces tan anchas como las de $M$. chilensis Lindl. (especie tipo), tépalos más cortos y apéndices bífidos y parduscos; cápsula con un diámetro de $10 \mathrm{~mm}$ y los cuernos puntiagudos de su base de 1,5 $\mathrm{mm}$ de largo, sin embargo sólo un espécimen con frutos fue depositado en el herbario SGO (abreviaciones sensu Holmgren et al. 1990). Posteriormente, Reiche (1893) reconoce a esta especie y menciona además que el tubo estaminal presenta dos protuberancias inclinadas que caen hacia delante. Recolecciones posteriores fueron escasas y por el desconocimiento de las características florales, algunos autores no la reconocen (Hutchinson 1939, Traub 1963, Marticorena \& Quezada 1985) o la consideraron como sinónimo de M. chilensis (Baker 1879).

Ravenna (2000) publicó una completa descripción de $M$. cornuta basado en ejemplares recolectados en localidades cercanas a la cuesta El Melón, sin embargo algunas de las características como tépalos reflexos y protuberancias (cuernos) basales de la cápsula, caracteres diagnósticos, no fueron consideradas, señalándose, por el contrario, un par de cuernos sobre los tres vértices apicales de la cápsula.

El año 2007 se observó en el Jardín Botánico Nacional una población natural muy pequeña de Miersia, que presentaba tépalos reflexos, urna o bolsa estaminal con pliegue y en el fruto, tres protuberancias basales en forma de cuernos (Novoa obs. pers.). Posteriormente, el año 2009 se recolectó material con flor y fruto en dicha localidad y en las quebradas de la cuesta El Melón, el cual se comparó con el material tipo de la especie y otros pocos ejemplares depositados en CONC y SGO, constatándose que se trataba de la especie descrita por R.A. Philippi en 1873.

Miersia cornuta Phil., Anales Univ. Chile 72: 548. 1873. TIPO: Chile. "en la cuesta de Melon, en la provincia de Aconcagua, que en setiembre de 1865 tenia ya el fruto casi maduro" (Holotipo: SGO 37944!).

DesCripción AMPLIADA: Plantas herbáceas, bulbosas. Bulbo ovoide, de 1-2 x 1-1,7 cm. Hojas 1-2 (-6), lineares, de 25$52 \mathrm{~cm}$ de largo x 2-5 mm de ancho. Escapo 1, de 21-42 $\mathrm{cm}$ de alto. Brácteas ovadas, herbáceas, de 0,8-1,2 cm de alto x 2-6 mm de ancho. Inflorescencia con 3-6 flores. Pedicelos desiguales, de $0,6-6,5 \mathrm{~cm}$ de largo. Tépalos 6 , oval-lanceolados o lanceolados, reflexos, verdes, a veces con líneas purpúreas, superficie interna papilosa; tépalos superiores, algo laciniados, con el nervio medio marcado, los externos superiores de 5-7 mm de largo x 1-1,8 mm de ancho, el externo inferior de 5,5 $\mathrm{mm}$ de largo x 1,5-2 $\mathrm{mm}$ de ancho; los tépalos internos de 5-6,5 $\mathrm{mm}$ de largo x $0,8-1 \mathrm{~mm}$ de ancho. Apéndices florales 6, filiformes, enteros, papilosos, purpúreos, de 1,4-2,5 $\mathrm{mm}$ de largo. Estambres 6, filamentos unidos formando el tubo, bolsa o urna estaminal quedando libres por dentro de ésta en su tercio superior, donde expansiones de éstos complementan la urna. Tubo estaminal deflexo, de 2-2,5 mm de largo, desigualmente ancho, con la parte basal inflada o globosa formando un pliegue que cae abruptamente sobre el mismo y es cilíndrico hacia la parte distal donde se separan los filamentos dejando visibles sólo las anteras, tubo estaminal generalmente de coloración verde con la parte 
cilíndrica purpúrea. Anteras, de 0,4-0,5 mm de largo, moradas previas a la antesis, posteriormente amarillas. Ovario súpero, esférico, trilocular. Estilo inclinado y estigma capitado. Cápsulas de 0,4-1 cm de largo x 0,4-1 $\mathrm{cm}$ de ancho, cada lado de contorno cuadrangular, con protuberancias basales en forma de cuernos, de 0,6-1,5 $\mathrm{mm}$ de largo, originadas por la unión del margen basal de cada lóculo del ovario. Semillas esféricas, castaño oscuras, de $0,8 \mathrm{~mm}$ de diámetro (Fig. 1).

Material estudiado: CHILE: Región de Coquimbo, Prov. Choapa. Tilama. 550 m, 26-VII-1938, C. Grandjot s.n. (CONC); Región de Valparaíso, Prov. Quillota. Palos Quemados, 400-700 m, 11-VIII-1935. C. Grandjot s.n. (CONC); Quebrada de Pucalán, Nogales, 10-VIII-2000. M.T. Eyzaguirre s.n. (SGO); Cuesta el Melón, quebradas

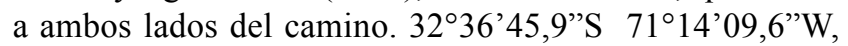
564 m, 13-VIII-2009. I. Escobar \& P. Novoa 182, 186 y 189 (CONC); Prov. Valparaíso. Jardín Botánico de Viña del Mar, sector laguna de Linneo. 33 02'20,40”S 71'29'48,72”W, 70 m, 13-VIII-2009. I. Escobar \& P. Novoa 177 (CONC).

DistRIBUCIÓN Y HÁBITAT: $M$. cornuta se distribuye por la cordillera de la Costa en las provincias de Choapa, Quillota y Valparaíso. Crece en taludes o cortes de terreno asociada a las quebradas, bajo vegetación nativa, principalmente Lithrea caustica (Molina) Hook. et Arn., Peumus boldus Molina, Quillaja saponaria Molina y Beilschmiedia miersii (Gay) Kostermans. Su floración ocurre entre los meses de julio a agosto.

OBSERVACIONES: Las estructuras florales en Miersia han sido interpretadas de forma diferente por varios autores. Particularmente en $M$. cornuta, los apéndices florales han sido señalados como hojuelas interiores persistentes y bífidas (Philippi 1873), lígulas bífidas (Reiche 1893), 6 pequeños apéndices angostos y bífidos (Muñoz \& Moreira 2000) y finalmente como 6 apéndices enteros (Ravenna 2000). En $M$. chilensis y $M$. tenuiseta Ravenna, estos apéndices florales corresponden a 6 estructuras divididas, las que pueden ser planas o filiformes, respectivamente. En $M$. leporina sólo se encuentran dos estructuras superiores, planas y lanceoladas, mientras que en $M$. cornuta los apéndices corresponden a 6 estructuras enteras y filiformes (Fig. 1 A-B, 2 C).

La falta de disponibilidad de los ejemplares depositados en el herbario privado de P.F. Ravenna no permiten corroborar sus observaciones, sin embargo la recolección de materiales en las localidades tipo señaladas por el autor nos han permitido reconocer algunas de las especies por él descritas.

M. chilensis y $M$. tenuiseta constituyen generalmente poblaciones abundantes, con un amplio rango de distribución entre las regiones de Valparaíso a La Araucanía, y entre las regiones de Valparaíso y Metropolitana, respectivamente, y se caracterizan por un número mayor de escapos y hojas y por los lados triangulares de las cápsulas (Fig. 2 D-E). Por el contrario, M. cornuta y M. leporina Ravenna forman poblaciones muy localizadas, con pocos individuos, entre las regiones de Coquimbo a Valparaíso, y Coquimbo a Metropolitana, respectivamente. Ambas especies se caracterizan por sus escapos robustos y cápsulas con lados cuadrangulares (Fig. 2 A, F-G). Aunque es común encontrar a $M$. cornuta creciendo junto a representantes de las otras especies de Miersia, ella se distingue claramente por la presencia de tépalos reflexos, 6 apéndices florales filiformes y enteros, tubo estaminal ancho basalmente, formando un pliegue que cae abruptamente sobre el misma (Fig. 1 y 2 B-C).

\section{Clave para identificar las especies de Miersia Lindley.}

1. Plantas generalmente con 1-2(-6) hojas. Escapos 1(2). Cápsulas de contornos laterales cuadrangulares, con protuberancias basales.

2. Tépalos reflexos. Tubo estaminal forma un pliegue basal que cae abruptamente sobre el mismo. Apéndices florales 6 , rodeando la urna estaminal, filiformes, enteros.

2'. Tépalos no reflexos. Tubo estaminal sin pliegue basal. Apéndices florales 2, superiores, planos, enteros o superficialmente divididos.

1'. Plantas generalmente con 3-7 hojas. Escapos 2-3(4). Cápsulas de contornos laterales triangulares, con protuberancias apicales.

3. Flores bilaterales, tépalos lineares, acuminados, frecuentemente torcidos en el ápice. Apéndices florales planos, levemente divididos o divididos hasta la mitad de su longitud.

3'. Flores con tendencia radial, tépalos lanceolados, agudos, ápice no torcido. Apéndices florales filiformes, divididos desde la base.

M. tenuiseta 
Gayana Bot. 67(1), 2010

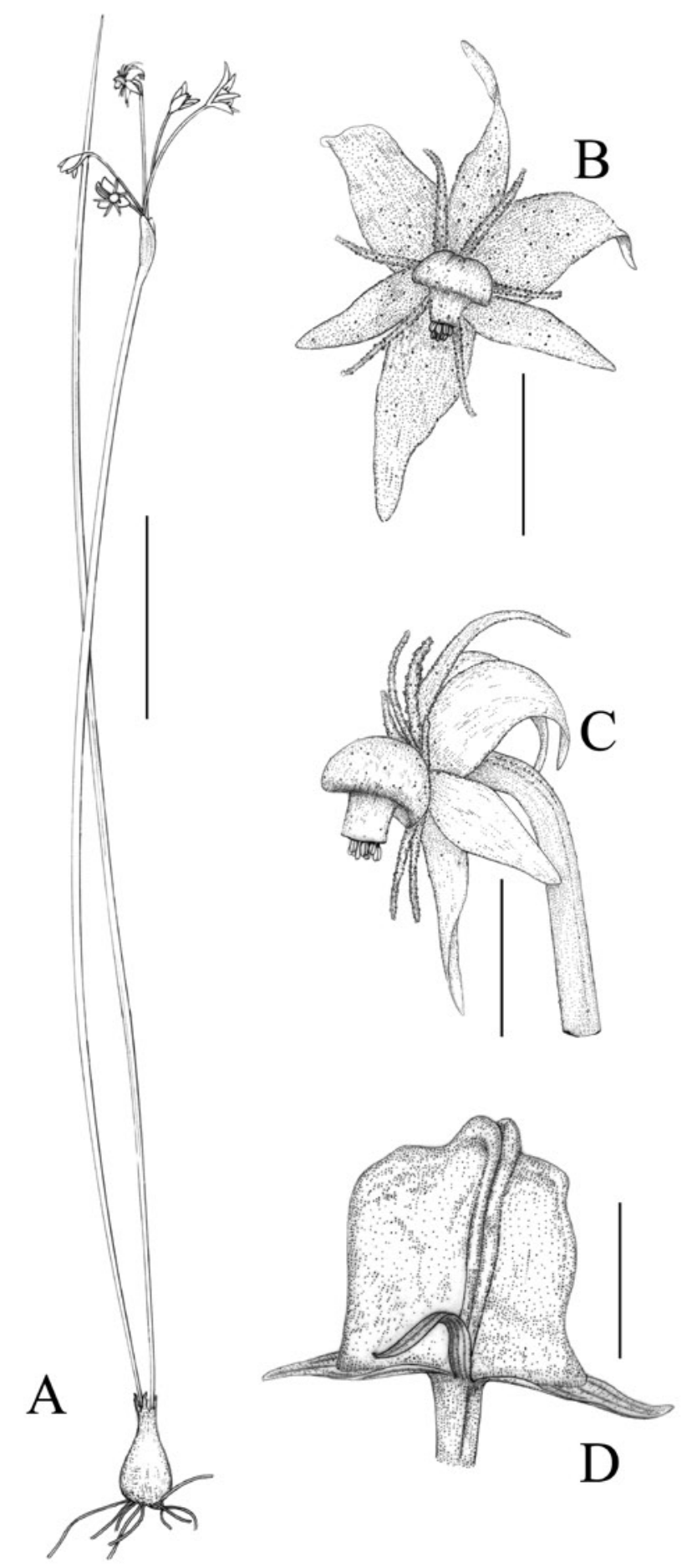

FIgURA 1. Ilustración de M. cornuta. (A) Hábito, (B) Flor vista frontal, estructuras filiformes corresponden a los seis apéndices enteros, (C) Detalle de la flor, mostrando tépalos reflexos, tubo estaminal ancho en la base y cilíndrico hacia las anteras, (D) Cápsulas triloculares con lados de contorno cuadrangular, con prolongaciones basales con forma de cuernos. Escalas $\mathrm{A}=5,7 \mathrm{~cm} ; \mathrm{B}-\mathrm{C}=0,4 \mathrm{~cm} ; \mathrm{D}=0,6 \mathrm{~cm}$.

Figure 1. Illustration of M. cornuta. (A) Habit, (B) Flower frontal view, filiform structures correspond to 6 floral appendages, (C) Details of flower, showing reflexed tepals, and deflexed staminal tube, (D) Capsules, each side with quadrangular outline, and basal horns. Bars $\mathrm{A}=5.7 \mathrm{~cm} ; \mathrm{B}-\mathrm{C}=0.4 \mathrm{~cm} ; \mathrm{D}=0.6 \mathrm{~cm}$. 
Miersia cornuta: EscoBAR, I. ET AL.
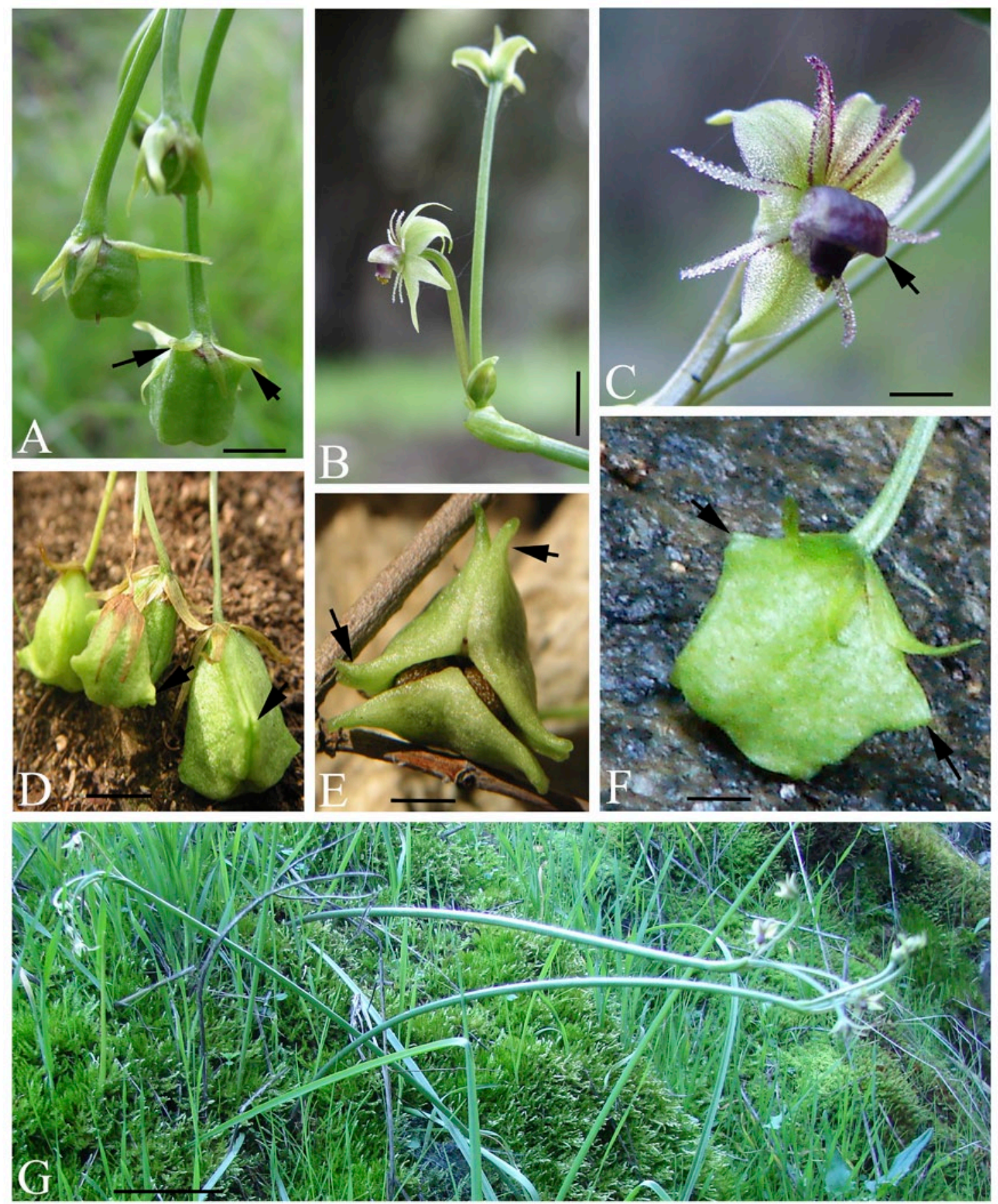

Figura 2. Fotografías de M. cornuta. (A) Cápsulas triloculares con lados de contorno cuadrangular, flechas indican posición de los cuernos. (B) Inflorescencia, vista lateral de la flor mostrando los tépalos reflexos. (C) Detalle de la flor, flecha indica la bolsa estaminal, estructuras filiformes corresponden a apéndices florales. (D y E). Frutos de M. chilensis, flechas indican la posición de las protuberancias apicales en forma de cuernos. (F) Detalle del fruto de M. cornuta, flechas indican las protuberancias basales con forma de cuernos. (G) Hábito de $M$. cornuta. Escalas: A, C y D=0,5 cm; $\mathrm{B}=0,8 \mathrm{~cm} ; \mathrm{E}=0,3 \mathrm{~cm} ; \mathrm{F}=0,2 \mathrm{~cm}$ y G $=6,5 \mathrm{~cm}$.

Figure 2. Photographs of $M$. cornuta. (A) Capsules of $M$. cornuta, each side with quadrangular outline, arrows indicates the horns position. (B) Inflorescence, lateral view of flower showing reflexed tepals. (C) Flower details, arrow indicates deflexed staminal tube, filiform structure corresponds to floral appendages. (D and E) Fruits of $M$. chilensis arrow indicates horns with apical position. (F) Fruit details of $M$. cornuta, arrow indicates basal horns. (G) Habit of $M$. cornuta. Bars: $\mathrm{A}, \mathrm{C}$ and $\mathrm{D}=0.5 \mathrm{~cm} ; \mathrm{B}=0.8 \mathrm{~cm} ; \mathrm{E}=0.3 \mathrm{~cm} ; \mathrm{F}=0.2$ $\mathrm{cm}$ and $\mathrm{G}=6.5 \mathrm{~cm}$. 
ESTADO DE CONSERVACIÓN: $M$. cornuta ha sido señalada como una especie en Peligro de Extinción, por su distribución restringida, pastoreo y alteración de la vegetación por incendios (Ravenna et al. 1998). El presente trabajo y de acuerdo a los criterios de la UICN versión 3.1 (2001), se considera como Vulnerable (VU), dado que se estima una extensión de la presencia geográfica menor de 20.000 $\mathrm{km}^{2}$, severamente fragmentada, con disminución inferida en la calidad del hábitat, debido al cultivo de frutales y al pastoreo de animales domésticos, además está constituida por poblaciones muy localizadas y poco abundantes, con un tamaño estimado menor a mil individuos maduros [VU: Blab(iii) + D1].

\section{AGRADECIMIENTOS}

A los curadores de los herbarios SGO y CONC, por las facilidades otorgadas para revisar dichas colecciones, al Dr. Roberto Rodríguez por la revisión del manuscrito y a Pedro Arias por la elaboración de la ilustración. A los proyectos Flora de Chile, DIUC 208.111.049.1-0 y Beca de apoyo a tesis (CONICYT).

\section{BIBLIOGRAFÍA}

BAKER, J.G. 1879. A synopsis of Colchicaceae and the Aberrant Tribes of Liliaceae. Journal of the Linnean Society Botany. 17: 405-510.

Holmgren, P.K., N.H. Holmgren \& L.C. Barnett. 1990. Index
Herbariorum. Part I: The herbaria of the world. Edition 8. New York Botanical Garden. 693 pp.

Hutchinson, J. 1939. The tribe Gilliesieae of Amaryllidaceae. Herbertia 6: 136-145.

Marticorena, C. \& M. Quezada. 1985. Catálogo de la flora vascular de Chile. Gayana Botánica 42: 1-157.

Muñoz, M. \& A. Moreira. 2000. Los géneros endémicos de Monocotiledóneas de Chile continental. URL:http:// www.chlorischile.cl/

Philippi, R.A. 1873. Descripción de las plantas nuevas incorporadas últimamente en el herbario chileno. Anales de la Universidad de Chile 43: 479-583.

Ravenna, P., S. Teillier, J. Macaya, R. Rodríguez \& O. Zöllner. 1998. Categorías de conservación de las plantas bulbosas nativas de Chile. Boletín del Museo Nacional de Historia Natural. 47: 47-68.

Ravenna, P. 2000. New or noteworthy Miersia species (Gilliesiaceae). Onira 5: 31-34.

ReIche, K. 1893. Beiträge zur Kenntnis der Liliaceae-Gilliesieae. Botanische Jahrbücher für Systematik 16: 262-277.

Traub, H. 1963. The genera of Amaryllidaceae. American Plant Life Society, La Jolla, California, USA. 85 pp.

UICN. 2001. Categorías y Criterios de la Lista Roja de la UICN: Versión 3.1. Comisión de Supervivencia de Especies de la UICN. Unión Internacional para la Conservación de la Naturaleza y Recursos Naturales, Gland, Suiza y Cambridge, Reino Unido. 33 pp.

Zuloaga, F. O., Morrone, O. \& M. Belgrano (Eds). 2008. Alliaceae. Catálogo de las Plantas Vasculares del Cono Sur (Argentina, Sur de Brasil, Chile, Paraguay y Uruguay). Pteridophyta, Gymnospermae, Monocotyledoneae. Monograph in Systematic Botany from Missouri Botanical Garden 107(1); i-xcvi, 1-983. 